\title{
Investigation on Asymmetric Parallel-coupled CPW for $\lambda / 4$ Bandpass Filters with Broad Rejection Band
}

\author{
Jing Gao ${ }^{1}$ and Lei $\mathbf{Z h u}^{1 \mathrm{a})}$ \\ ${ }^{1}$ School of Electrical \& Electronic Engineering, Nanyang Technological University, \\ Nanyang Avenue, Singapore 639798 \\ a)ezhul@ntu.edu.sg
}

Abstract: Asymmetric parallel-coupled coplanar waveguide (CPW) is originally characterized here as an equivalent unified K-inverter network. On a basis of our developed self-calibrated method of moments (MoM), the relevant network parameters are extracted to explicitly demonstrate the frequency-dependent coupling behavior with the $1^{\text {st }}$ zero coupling at the $\lambda / 2$ length of overlapped section. By differentiating the coupled spaces and overlapped lengths, the two CPW coupling structures are dissimilarly constructed and utilized as the input/output excitation of a $\lambda / 4 \mathrm{CPW}$ bandpass filter. Through allocating the two unequal zeros towards the spurious harmonic around $3 \lambda / 4$, an improved two-stage $\lambda / 4 \mathrm{CPW}$ filter is designed at $5.0 \mathrm{GHz}$. Optimized results achieve broad rejection band, ranging from $5.8 \mathrm{GHz}$ beyond $18.0 \mathrm{GHz}$, as verified by the ADS simulation.

Keywords: asymmetric parallel-coupled CPW, K-inverter, $\lambda / 4$ bandpass filter, harmonic suppression, and self-calibrated method of moments.

Classification: microwave millimeter-wave devices, circuits and systems

\section{References}

[1] D. F. Williams and S. E. Schwarz, "Design and performance of coplanar waveguide bandpass filters," IEEE Trans. Microwave Theory Tech., vol. 31, no. 7, pp. 558-566, July 1983.

[2] J. K. A. Everard and K. K. M. Cheng, "High performance direct coupled bandpass filters," IEEE Trans. Microwave Theory Tech., vol. 41, no. 9, pp. 1568-1573, Sep. 1993.

[3] T. Tsujichi, H. Matsumoto, and T. Nishikawa, "A miniaturized endcoupled bandpass filter using $\lambda / 4$ hair-pin coplanar resonators," 1998 IEEE MTT-S Int. Microwave Symp. Dig., pp. 829-302.

[4] A. Sanada, H. Takehara, T. Yamamoto, and I. Awai, " $\lambda / 4$ steppedimpedance resonator bandpass filters fabricated on coplanar waveguide," 2002 IEEE MTT-S Int. Microwave Symp. Dig., pp. 385-388.

[5] W. Menzel, W. Schwab, and G. Strauss, "Investigation of coupling structures for coplanar bandpass filters," 1995 IEEE MTT-S Int. Microwave 
Symp. Dig., pp. 1407-1410.

[6] T. M. Weller, "Edge-coupled coplanar waveguide bandpass filter design," IEEE Trans. Microwave Theory Tech., vol. 48, no. 12, pp. 2453-2458, Dec. 2000.

[7] T. Paillot, P. Blondy, D. Cros, and P.-Y. Guillon, "A novel compact filter," 2002 IEEE MTT-S Int. Microwave Symp. Dig., pp. 1793-1796.

[8] L. Zhu and K. Wu, "Accurate circuit model of interdigital capacitor and its application to design of new quasi-lumped miniaturized filters with suppression of harmonic resonance," IEEE Trans. Microwave Theory Tech., vol. 48, no. 3, pp. 347-356, Mar. 2000.

[9] L. Zhu and T. Yakabe, "Fullwave MoM-SOC technique for extraction of equivalent circuit models coplanar waveguide discontinuities: $\mathrm{CPW}$ - and CSL-mode cases," IEICE Trans. Electron., vol. E86-C, no. 11, pp. 22922299, Nov. 2003.

\section{Introduction}

Coplanar waveguide (CPW) bandpass filters [1-7] have been extensively studied in the design of uniplanar microwave integrated circuits and subsystems. In $[1,2]$, CPW capacitive or inductive gap structures were initially utilized as lumped coupling elements to make up the two initial half-wavelength $(\lambda / 2)$ CPW bandpass filters. By stagger excitations of $\lambda / 4$ CPW resonators with these two coupling elements, the two $\lambda / 4$ CPW filters [3, 4] were developed with size-compactness and $1^{\text {st }}$ harmonic suppression. But, due to very weakly coupling degree of these lumped structures, constituted CPW filters usually suffer from high insertion loss and narrow pass bandwidth, especially at low frequency as reported in [1-2, 4].

By parallel-overlapping the two CPW short-ends with unequal strip/slot widths, an asymmetric parallel-coupled CPW structure was originated in [5] to strengthen the concerned coupling degree and further applied to build up the compact $\lambda / 4$ and $\lambda / 2 \mathrm{CPW}$ filters [5-7] with low insertion loss and wide dominant passband. So far, this distributed coupling structure was limitedly modeled via two-dimensional spectral domain approach [6] to approximately expose its coupling behavior under the ideal $\lambda / 4$ overlapped length in terms of even- and odd-mode characteristic impedances. No reported work has been carried out today to demonstrate its overall frequency-dependent coupling characteristics.

In this work, our effort concentrates itself to characterize this distributed CPW coupling structures into its equivalent K-inverter network via our selfcalibrated method of moments, namely, MoM-SOC [8, 9]. Extensive results demonstrate that the relevant coupling degree, i.e., K-impedance, varies as a quasi-sinusoidal function of frequency while its value becomes zero at the frequency with the $\lambda / 2$ overlapped length. With the use of the two dissimilar CPW coupling elements at the input/output ports, an improved $\lambda / 4$ CPW filter with broad upper rejection band is designed by allocating the two unequal coupling zeros to the $2^{\text {nd }}$ harmonic. 


\section{Asymmetric Parallel-Coupled CPW}

The two CPW short-ends with different strip widths ( $\mathrm{w}_{1}$ and $\mathrm{w}_{2}$ ) are overlapped in a parallel way as indicated in Fig. 1 (a) to make up a distributed CPW inductive coupling structure [5]. Following the work in [8], this asymmetric CPW structure with two dissimilar CPW feeders can be accordingly expressed as an equivalent $\mathrm{K}$-inverter network with the K-impedance $(K)$ and two unequal electrical lengths at its two sides $\left(\phi_{1} / 2\right.$ and $\left.\phi_{2} / 2\right)$. As detailed in [9], such a two-port CPW element is at first numerically characterized as its Z-matrix with three independent reactances, i.e., $X_{11}, X_{12}=X_{21}$ and $X_{22}$, by executing our developed MoM-SOC program. Under the network equivalence [8], the three K-network parameters can be deduced as,

$$
\begin{aligned}
& \phi_{1}=\arctan \frac{2\left(\bar{X}_{11}+\bar{X}_{22}|\bar{X}|\right)}{\left(1+\bar{X}_{22}^{2}-\bar{X}_{11}^{2}-|\bar{X}|^{2}\right)}+k_{1} \pi \\
& \phi_{2}=\arctan \frac{2\left(\bar{X}_{22}+\bar{X}_{11}|\bar{X}|\right)}{\left(1+\bar{X}_{11}^{2}-\bar{X}_{22}^{2}-|\bar{X}|^{2}\right)}+k_{2} \pi \\
& \frac{K}{\sqrt{Z_{1} Z_{2}}}=\frac{-\sin \frac{\phi_{1}}{2}+\bar{X}_{11} \cos \frac{\phi_{1}}{2}}{\bar{X}_{12} \sin \frac{\phi_{2}}{2}}=\frac{\bar{X}_{12} \cos \frac{\phi_{2}}{2}}{X_{11} \sin \frac{\phi_{1}}{2}+\cos \frac{\phi_{1}}{2}}
\end{aligned}
$$

in which $\bar{X}_{11}=X_{11} / Z_{1}, \bar{X}_{22}=X_{22} / Z_{2}$ and $\bar{X}_{12}=X_{12} / \sqrt{Z_{1} Z_{2}}$ are the

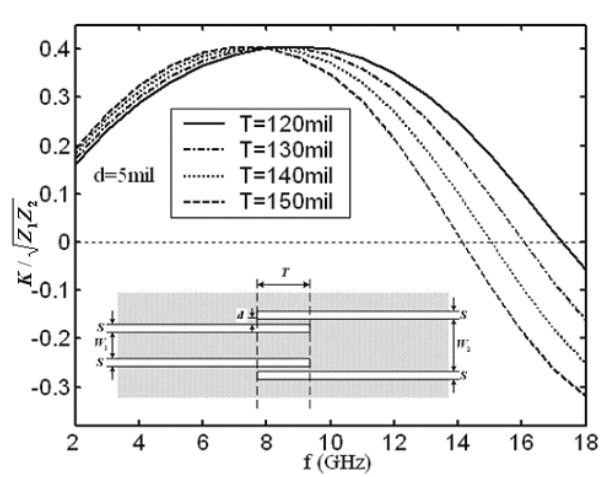

(a)

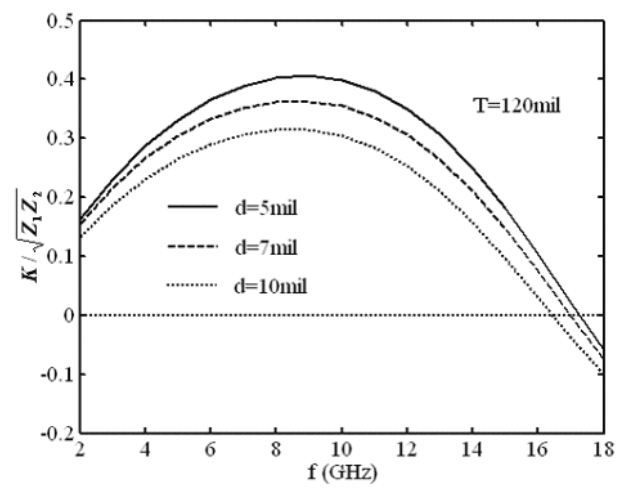

(c)

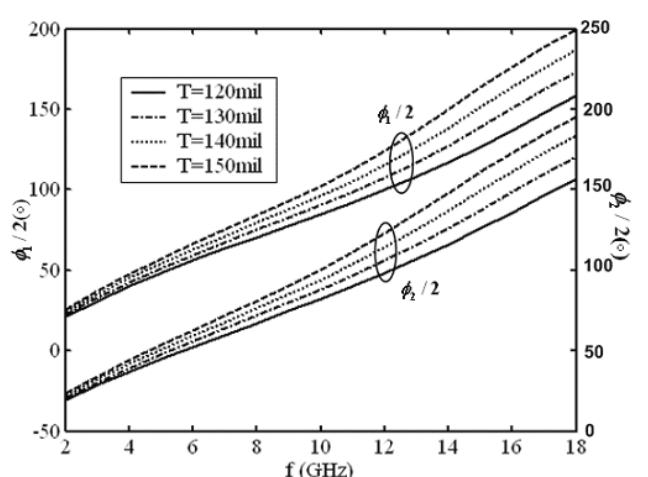

(b)

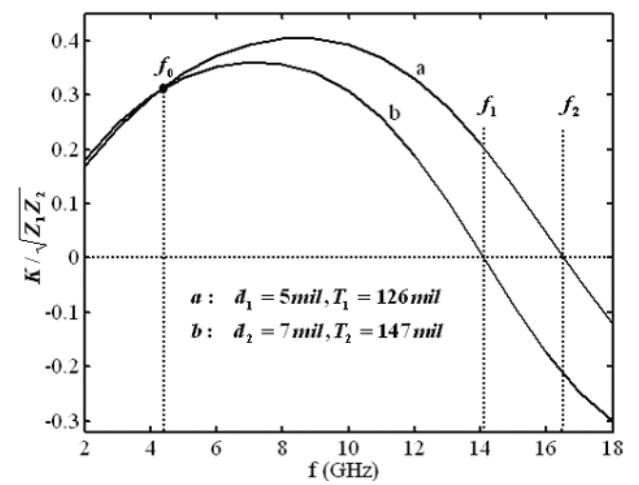

(d)

Fig. 1. K-inverter network parameters of the paralleloverlapped CPW structure. (a), (c), (d) normalized K-impedance; (b) equivalent electrical lengths. 
relevant normalized reactances with respect to characteristic impedances, $\mathrm{Z}_{1}$ and $\mathrm{Z}_{2}$, of the two CPW feeders. $|\bar{X}|=\bar{X}_{11} \bar{X}_{22}-\bar{X}_{12}^{2}$ while $k_{1}$ and $k_{2}$ are the integer.

Fig. 1 (a) depicts the extracted K-inverter impedance versus overlapped length ( $\mathrm{T}$ ) as a function of frequency (f). As frequency increases, the Kimpedance is observed to gradually go up to its maximum and then fall down across the zero point, thereby exhibiting the quasi-sinusoidal frequency dispersion. Furthermore, we can find that this transmission zero is moved down as the overlapped length (T) is extended. Fig. 1 (b) shows the two relevant electrical lengths versus frequency. It can be seen here that the K-impedance basically achieves its maximum around the frequency where $\phi_{2} / 2=90^{0}$ while gets to the zero minimum when $\phi_{2} / 2=180^{\circ}$. Fig. 1 (c) describes the normalized K-impedance versus the space (d) between the overlapped-CPW slots. K-impedance curve tends to decrease with $\mathrm{d}$ since the coupling between the two $\mathrm{CPW}$ is reduced. In order to build up a $\lambda / 4 \mathrm{CPW}$ filter with harmonic suppression in the next section, the two dissimilar CPW coupling structures with narrow and wide spaces, i.e., $\mathrm{d}_{1}$ and $\mathrm{d}_{2}$, are further characterized to achieve the unchanged coupling degree at the central frequency $\left(\mathrm{f}_{0}\right)$ of the dominant passband while allocate the two transmission zeros to $f_{1}$ and $f_{2}$, respectively, around the $2^{\text {nd }}$ harmonic passband. It can be realized by extending the CPW overlapped length with $\mathrm{d}_{2}$ as illustrated in Fig. 1 (d).

\section{Design Example: Improved $\lambda / 4$ CPW Filter}

Fig. 2 (a) depicts the proposed $\lambda / 4 \mathrm{CPW}$ bandpass filter. The two $\lambda / 4$ CPW resonators with unequal strip/slot widths are inductively excited by the two parallel-coupled CPW with different spaces $\left(\mathrm{d}_{1}\right.$ and $\left.\mathrm{d}_{2}\right)$ and overlapped length $\left(\mathrm{L}_{1}\right.$ and $\left.\mathrm{L}_{5}\right)$ while they are capacitively coupled together via gap width $\left(\mathrm{L}_{3}\right)$. Fig. $2(\mathrm{~b})$ indicates the relevant equivalent cascaded topology with the pre-calculated two K-impedance $\left(\mathrm{K}_{1}, \mathrm{~K}_{2}\right)$ and one J-susceptance $(\mathrm{J})$ via MoM-SOC as well as the two $\lambda / 4$ transmission line resonators at the central frequency.

Fig. 3 (a) illustrates the three sets of calculated insertion losses $\left(\mathrm{S}_{21}\right)$ in conjunction with the three cases. For the traditional $\lambda / 4$ CPW filter without adjusting the overlapped length [4] at both CPW coupling elements, the spurious harmonic appears around 13.6 to $16.8 \mathrm{GHz}$ with two peaks as observed in the solid curve. By suitably adjusting the overlapped length to $\mathrm{L}_{1}=\mathrm{L}_{5}=147$ mil under the other dimensions of $\mathrm{d}_{1}=\mathrm{d}_{2}=7 \mathrm{mil}, \mathrm{L}_{2}=\mathrm{L}_{4}=80 \mathrm{mil}$ and $\mathrm{L}_{3}=28 \mathrm{mil}$, the single transmission zero can be observed and it is suitably allocated to $13.8 \mathrm{GHz}$, thereby canceling the $1^{\text {st }}$ peak as shown in the dashed curve. Following the description in Fig. 1(d), the two asymmetric CPW structures with $\mathrm{d}_{1}=5 \mathrm{mil}, \mathrm{d}_{2}=7 \mathrm{mil}, \mathrm{L}_{1}=126 \mathrm{mil}$ and $\mathrm{L}_{5}=147 \mathrm{mil}$ are formulated to simultaneously bring out the two transmission zeros targeting the two separate peaks, i.e., $13.8 \mathrm{GHz}$ and $16.2 \mathrm{GHz}$. As exhibited in the dotted line in Fig. 3(a), both peaks are consequently canceled out so as to effectively suppress this spurious harmonic passband. 


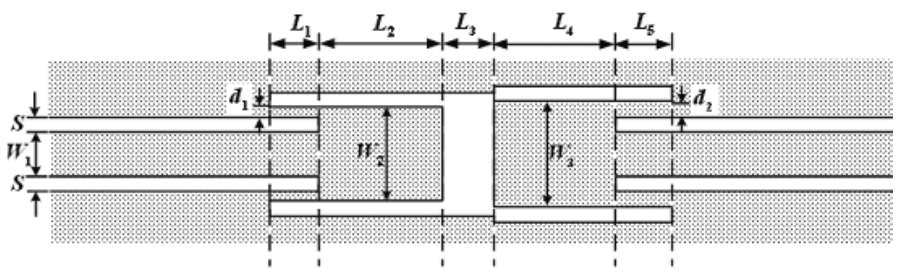

(a)

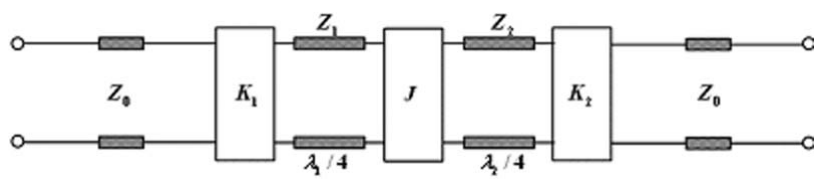

(b)

Fig. 2. The proposed two-stage CPW $\lambda / 4$ bandpass filter. (a) physical layout; (b) equivalent circuit topology.

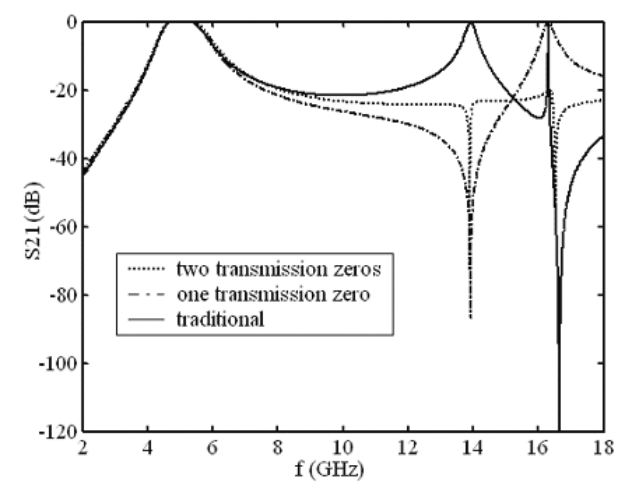

(a)

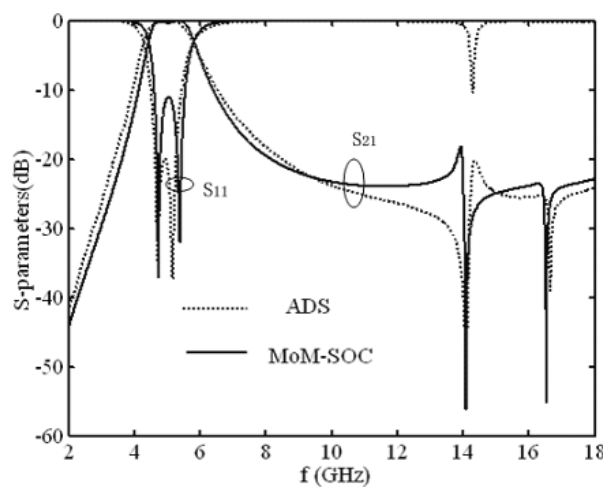

(b)

Fig. 3. Derived S-parameters of the proposed $\lambda / 4 \mathrm{CPW}$ bandpass filters. (a) three sets of results for physical understanding; (b) optimized results with ADS-simulation.

(Filter specifications: $\mathrm{f}_{0}=5.0 \mathrm{GHz}$ and $\mathrm{BW}=$ $28 \%$;substrate: $\varepsilon \boldsymbol{r}=10.2$ and $\mathrm{h}=25 \mathrm{mil}$; dimensions: $\mathrm{d}_{1}=5 \mathrm{mil}, \mathrm{d}_{2}=7 \mathrm{mil}, \mathrm{L}_{1}=126 \mathrm{mil}, \mathrm{L}_{2}=99 \mathrm{mil}$, $\mathrm{L}_{3}=25$ mil, $\mathrm{L}_{4}=80$ mil and $\mathrm{L}_{5}=147 \mathrm{mil}$ )

Fig. 3 (b) depicts the optimized results as comparison to the direct ADS simulation. Both curves illustrate the two transmission poles within the dominant passband of the fractional bandwidth of $\mathrm{BW}=28 \%$ at the central frequency of $\mathrm{f}_{0}=5.0 \mathrm{GHz}$. Meanwhile, the upper rejection band is significantly broadened to the range from $5.8 \mathrm{GHz}$ beyond $18.0 \mathrm{GHz}$ relying on the separate allocation of the two transmission zeros at the frequencies of $14.0 \mathrm{GHz}$ and $16.6 \mathrm{GHz}$. 


\section{Conclusions}

This paper presents an effective method for spurious harmonic suppression in the CPW bandpass filter design by using the two asymmetric parallel-coupled CPW at the input/output ports. Such a distributed coupling structure is modeled as a unified K-inverter network so as to quantitatively demonstrate its frequency-dependent coupling behavior in a quasi-sinusoidal-function variation. Detailed discussion is made to exhibit its transmission zero as a function of overlapped length and further allocate the two zeros to suppress the $2^{\text {nd }}$ spurious harmonic. Optimized results on a proposed two-stage $\lambda / 4 \mathrm{CPW}$ bandpass filter show us an ultra-broad rejection band with simultaneous cancellation of the $1^{\text {st }}, 2^{\text {nd }}$ and harmonic passbands as confirmed by the ADSsimulated results. 